\title{
Augmentation of Macular Pigment following Implantation of Blue Light-Filtering Intraocular Lenses at the Time of Cataract Surgery
}

\author{
Jobn M. Nolan, ${ }^{1}$ Philip O'Reilly, ${ }^{1}$ James Loughman, ${ }^{2}$ Jim Stack, ${ }^{1}$ Edward Loane, ${ }^{1}$ \\ Eithne Connolly, ${ }^{1}$ and Stephen Beatty ${ }^{1}$
}

\begin{abstract}
Purpose. (Photo)-oxidative stress is believed to play a role in the pathogenesis of age-related macular degeneration (AMD), with the threshold for retinal damage being lowest for shortwavelength (blue) light. Macular pigment (MP), consisting of the carotenoids lutein $(\mathrm{L})$, zeaxanthin $(\mathrm{Z})$ and meso-Z, has a maximum absorption at $460 \mathrm{~nm}$ and protects the retina from (photo)-oxidative injury. This study was designed to investigate whether the blue light-filtering properties of the Alcon AcrySof Natural intraocular lens (ANIOL) implanted during cataract surgery affects MP optical density (MPOD).
\end{abstract}

Methods. Forty-two patients scheduled for cataract surgery were recruited for the study. These patients all had a preoperative best corrected visual acuity rating (BCVAR) of at least 0.5 (logMAR) in the study eye. The patients were randomized to have either the standard Alcon AcrySof three-piece acrylic intraocular lens (AIOL) (controls) or the ANIOL implanted at the time of cataract surgery. The spatial profile of MPOD (i.e., at $0.25^{\circ}, 0.5^{\circ}, 1.0^{\circ}$, and $1.75^{\circ}$ eccentricity) was measured with customized heterochromatic flicker photometry (cHFP) 1 week before and 1 week after surgery, and at 3, 6, and 12 months after surgery. Serum concentrations of $\mathrm{L}$ and $\mathrm{Z}$ were also measured at each study visit.

Results. There was a highly significant and positive correlation between all MPODs (e.g., at $0.25^{\circ}$ ) recorded 1 week before and after surgery in eyes with an AIOL implant $(r=0.915, P<$ 0.01 ; paired samples $t$-test, $P=0.631$ ) and in those ANIOL implants $(r=0.868, P<0.01$; paired samples $t$-test, $P=$ $0.719)$. Average MPOD across the retina increased significantly with time (after 3 months) in the ANIOL group (repeatedmeasures, general linear model, $P<0.05$ ), but remained stable in the AIOL group (repeated-measures, general linear model, $P>0.05)$. There were no significant time or lens effects observed for serum $L$ over the study period $(P>0.05)$. There was a significant time effect for serum $\mathrm{Z}$ over the study period

From the ${ }^{1}$ Macular Pigment Research Group, Department of Chemical and Life Sciences, Waterford Institute of Technology, Waterford, Ireland; and the ${ }^{2}$ Macular Pigment Research Group, Optometry Department, School of Physics, Dublin Institute of Technology, Dublin, Ireland.

Supported in full by Alcon Laboratories Inc., Fort Worth, Texas. Submitted for publication December 8, 2008; revised March 18, 2009; accepted August 21, 2009.

Disclosure: J.M. Nolan, Alcon Laboratories Inc. (F); P. O'Reilly, Alcon Laboratories Inc. (F); J. Loughman, Alcon Laboratories Inc. (F); J. Stack, Alcon Laboratories Inc. (F); E. Loane, Alcon Laboratories Inc. (F); E. Connolly, Alcon Laboratories Inc. (F); S. Beatty, Alcon Laboratories Inc. $(\mathrm{F})$

The publication costs of this article were defrayed in part by page charge payment. This article must therefore be marked "advertise$m e n t "$ in accordance with 18 U.S.C. $\$ 1734$ solely to indicate this fact.

Corresponding author: John M. Nolan, Macula Pigment Research Group, Waterford Institute of Technology, Cork Road, Waterford, Ireland; jnolan@wit.ie.
$(P<0.05)$, but not a significant time/lens interaction $(P>$ $0.05)$.

Conclusions. Customized HFP can reliably measure the MPOD spatial profile in the presence of lens opacity, and cataract surgery does not artifactually alter MPOD readings. This study also provides evidence that implanting an IOL that filters blue light is associated with augmentation of MPOD in the absence of raised serum concentrations of $\mathrm{L}$ and $\mathrm{Z}$. However, further and longitudinal study is needed to assess whether the observed increase in MPOD after implantation of blue-filtering IOLs is associated with reduced risk of AMD development and/or progression. (Invest Ophthalmol Vis Sci. 2009;50: 4777-4785) DOI:10.1167/iovs.08-3277

A ge-related macular degeneration (AMD), which damages central vision, is the most common cause of age-related blindness in the western world. ${ }^{1,2}$ Although the pathogenesis of AMD remains unclear, there is a growing body of evidence suggesting that oxidative stress is important in the pathogenesis of this condition and that cumulative short-wavelength (blue) light damage plays a role..$^{3-5}$

Macular pigment (MP), which is entirely of dietary origin, and composed of the xanthophyll carotenoids: lutein $(\mathrm{L})$, zeaxanthin $(\mathrm{Z})$, and meso- $\mathrm{Z}$, is thought to protect against AMD because it absorbs short-wavelength (blue) light at a prereceptoral level and because of its antioxidant properties. ${ }^{6,7}$ The absorption spectrum of MP peaks at $460 \mathrm{~nm}$ and may therefore limit photo-oxidative damage to retinal cells. ${ }^{8}$ MP levels are maximum within the photoreceptor axons of the foveola and the plexiform layers of the macula. ${ }^{6,9}$ Of importance, both the absorptive characteristics of MP and its location in the anterior portion of individual photoreceptors enables the pigment to attenuate the amount of blue light incident on the photoreceptor.

It has been hypothesized that cataracts provide protection against AMD by absorbing blue light, and thus reducing photooxidative damage to the retina. ${ }^{10}$ However, this protective effect, if any, would be restricted to certain types of lens opacity, such as nuclear sclerosis. In contrast, however, some studies have shown increased risk of cataract in association with AMD, which may reflect the fact that these conditions share antecedents (such as age). ${ }^{11-13}$ The positive association of AMD and cataract is considered to be an effect of similar causation and risk factors of both disorders. Although some studies have failed to find a link between cumulative sunlight exposure and the risk of development of AMD ${ }^{14-16}$ many other studies have found a positive association between lifetime exposure to sunlight and AMD.$^{17-20}$ Recently, the agerelated maculopathy and macular degeneration in elderly European populations (EUREYE) study has provided evidence of a link between cumulative (lifetime) sunlight exposure (in the presence of low antioxidant levels) and the risk of AMD. ${ }^{21}$ Those individuals with high cumulative lifetime exposure to sunlight but who were in the lowest quartile for combined antioxidant levels (especially vitamin $\mathrm{C}$, zeaxanthin, vitamin $\mathrm{E}$ 
and dietary zinc) were observed to have elevated odds ratios for AMD. ${ }^{21}$

Cataract surgery, where the natural crystalline lens is replaced with a clear artificial intraocular lens (IOL), has been shown to be an independent risk factor for the development or progression of AMD. ${ }^{13,22}$ There is increased short-wavelength light transmission to the retina after cataract surgery ${ }^{23,24}$ and an induced short-term intraocular inflammatory response (normally dampened by topical steroid administration), and either or both of these effects could enhance the risk of AMD progression and/or development. Indeed, ophthalmologists often observe the progression to advanced AMD shortly after cataract surgery. ${ }^{25}$ These observations have prompted lens manufacturers to incorporate a blue-light filter into the intraocular lens, in an attempt to attenuate photo-oxidative retinal injury and thereby reduce the risk of AMD development and/or progression.

Alcon has been producing a yellow (blue-light filtering) IOL, the Alcon AcrySof Natural SN60AT (ANIOL) since 2000. The ANIOL is similar to the standard, and commonly used, AcrySof SA60AT single-piece acrylic IOL (AIOL); however, it has a blue-light filtering capacity. The ANIOL was one of the first foldable IOLs to imitate the transmittance of the natural crystalline lens by combining a UV blocker with a covalently bound chromophore that partly absorbs light in the 400- to 500-nm spectral range. This lens is designed to simulate the light transmission characteristics of the adult non-cataractous human crystalline lens.

This study was designed to test the effect of the standard AIOL implant compared with the ANIOL implant on MP optical density (MPOD) by measuring MP at baseline (soon before and after implantation), and 3, 6, and 12 months after implantation.

\section{Methods}

\section{Patients and Randomization}

Forty-two patients scheduled for cataract surgery at Waterford Regional Hospital (WRH), Ireland, were recruited for the study, which was approved by the local Research Ethics Committee at WRH before study commencement. Informed written consent was obtained from each patient, and the experimental procedures adhered to the tenets of the Declaration of Helsinki.

Patients with a preoperative logMAR visual acuity of less than 0.5 (the minimum required for reliable measurement of MPOD) and those with any evidence of macular disease were not recruited. MPOD was measured 1 week before and 1 week after surgery, and at 3, 6, and 12 months after surgery. All study visits (five in total) were performed at the Macular Pigment Research Group's vision science laboratory at the Waterford Institute of Technology. The following details were recorded for each patient at baseline: lens prescription; general health status; tobacco use; body mass index (BMI) (defined as kilograms body weight/square meters height); ethnic background; skin color; iris color; and dietary assessment. Dietary assessment was also performed at the final visit. At all study visits (including baseline) the following study outcome measures were assessed: best corrected logMAR visual acuity; serum concentrations of $\mathrm{L}$ and $\mathrm{Z}$ (used to identify and control for any significant dietary and/or lifestyle changes); and MPOD, including its entire spatial profile across the retina.

Surgery was performed at WRH, and all patients had a clear corneal incision, continuous curvilinear capsulorrhexis, phacoemulsification, and in-the-bag IOL implantation. The patients were randomized to receive either the ANIOL or the AIOL implant at the time of surgery (in place of the cataractous crystalline lens). The trial was conducted in a double-blind, randomized, controlled fashion.

\section{Dietary Assessment}

Dietary assessment was performed at baseline and at the final study visit. A crude indicator of dietary intake and bioavailability of $\mathrm{L}$ and $\mathrm{Z}$ was constructed according to the frequency of consumption of five food items (dark green leafy vegetables, colored fruits and vegetables, eggs, fish, and overall fat intake) with examples given. The frequency of consumption was scored as follows: 0 , less than once a week; 1 , once a week; 2 , two to three times per week; 3 , four to six times per week; 4, once a day; 5 , more than once a day. Dietary fat intake (e.g., fried foods, snack foods, cheese, foods cooked in butter) was assessed due to its role in carotenoid absorption from the gut (fat intake was scored from 1 to 5 , as just outlined) ${ }^{26,27}$; fish intake was assessed due to its high concentration of $n-3$ docosahexaenoic acid, which has been shown to influence MP concentration (fish intake was also scored from 1 to 5 , as just outlined). ${ }^{28}$ In this way, an aggregate score for all food items was assigned to each person that ranged from 0 to 25 . The main purpose of assessing a person's dietary intake was to allow for adjustment of dietary confounding factors when performing statistical comparisons with other variables (e.g., lens type and MPOD).

\section{Serum Carotenoid Assessment}

Blood samples $(6-8 \mathrm{~mL})$ were collected from all patients on the same day as the dietary and MPOD assessment. Serum was separated from blood by centrifugation at $5000 \mathrm{rpm}$ for 10 minutes and then aliquoted into two light-sensitive microcentrifuge tubes and stored at minus $70^{\circ} \mathrm{C}$ until the time of analysis. Duplicate extractions were performed for each serum sample. A $400-\mu \mathrm{L}$ aliquot of serum was pipetted into a light-sensitive microcentrifuge tube $(1.5 \mathrm{~mL}$ total capacity). Ethanol $(300 \mu \mathrm{L})$ containing $0.25 \mathrm{~g} / \mathrm{L}$ butylated hydroxytoluene (BHT) and 200 $\mu \mathrm{L}$ internal standard ( $\alpha$-tocopherol acetate) was added to each tube. Heptane $(500 \mu \mathrm{L})$ was then added, and samples were vortexed vigorously for 1 minute followed by centrifugation at $2000 \mathrm{rpm}$ for 5 minutes (MSC Micro Centaur; Davison \& Hardy Ltd., Belfast, UK). The resulting heptane layer was retained and transferred to a second labeled light-sensitive microcentrifuge tube, and a second heptane extraction was performed. The combined heptane layers were immediately evaporated to dryness under nitrogen. These dried samples were reconstituted in $200 \mu \mathrm{L}$ methanol (containing $0.25 \mathrm{~g} / \mathrm{L} \mathrm{BHT}$ ), and 100 $\mu \mathrm{L}$ was injected for high-performance liquid chromatography (HPLC) analysis.

We used an HPLC (1200 series; Agilent Technologies Ltd., Dublin, Ireland) system with photodiode array detection. A 5- $\mu \mathrm{m}$ analytical/ preparative $4.6 \times 250-\mathrm{mm}$ specialty reversed-phase column (201TP; Vydac, Hesperia, CA) was used with an in-line guard column. The mobile phase consisted of $97 \%$ methanol and 3\% tetrahydrofuran. The flow rate was $1 \mathrm{~mL} / \mathrm{min}$, and the total run time was 13 minutes. All carotenoid peaks were integrated and quantified (ChemStation software; Agilent).

DSM Nutritional Products (Basel, Switzerland) provided the $\mathrm{L}$ and $\mathrm{Z}$ standards, which were used to generate standard curves for quantification of these carotenoids. This assay was validated against the National Institute of Standards and Technology (NIST) standards before analysis.

\section{Macular Pigment Measurement}

Apparatus. We used a macular densitometer, developed and originally described by Wooten et al., ${ }^{29}$ to measure MPOD, including its spatial profile across the retina. The densitometer uses heterochromatic flicker photometry (HFP) to obtain a valid measure of MPOD at a given retinal location. ${ }^{30}$

Procedure. The patient viewed a stimulus that alternated between a wavelength band absorbed by MP and one that was not. Test stimuli were presented in natural view and near the center of a $6^{\circ}$, $2.75-\mathrm{cd} / \mathrm{mm}^{2}, 470-\mathrm{nm}$ circular background. The patient adjusted the radiance of the wavelength band absorbed by MP to minimize (or eliminate) their perception of flicker. The range of alternation rates where flicker is not perceived is called the null zone. For the $460-\mathrm{nm}$ condition (maximum MP absorption), measurements at $0.25^{\circ}, 0.5^{\circ}, 1^{\circ}$, and $1.75^{\circ}$ eccentricity were obtained along the horizontal meridian of the nasal retina (for the left eye) and temporal retina (for the right eye) 
relative to a reference location at $7^{\circ}$ eccentricity. For the measures at $0.25^{\circ}$ and $0.5^{\circ}$ eccentricities, test stimuli were solid disks with those radii, with a small black fixation dot in the center. With these stimuli, when the flicker frequency is optimized, the edge hypothesis holds true up to eccentricities of approximately $2^{\circ} \cdot{ }^{31-33}$ For the $1^{\circ}$ and $1.75^{\circ}$ eccentricities, centrally fixated, 20-minute wide annuli with mean radii corresponding to those eccentricities were used. For the $7^{\circ}$ reference target, the test stimulus ( $2^{\circ}$ diameter disc) was viewed eccentrically using a small red light-emitting diode as a fixation target. The order of stimulus presentation was as follows: $0.5^{\circ}, 7^{\circ}, 1^{\circ}, 1.75^{\circ}$, and $0.25^{\circ}$.

For each stimulus condition/location, patients usually made five judgments of null flicker. However, there were patients for whom the initial, predicted HFP frequency value was either too high or too low. In these situations, the experimenter adjusted the frequency of the flickering stimulus in steps of $1 \mathrm{~Hz}$, either increasing it (if the patient could not eliminate flicker), or decreasing it (if the patient exhibited a wide range of null flicker values). The range of null flicker was considered too wide if the patient provided values that spanned a range of more than $\sim 15 \%$ radiance units $(\sim 0.07$ MPOD).

Customized HFP. Efforts were made in this study to optimize and customize the method, to facilitate obtaining MP measurements and ensure their accuracy. Similar techniques have been used in recently published studies in which MP was measured with the macular densitometer. ${ }^{34,35}$ Primarily, it is optimal to customize the HFP task for each patient (because of interindividual differences in flicker sensitivity) by selecting the alternation rate to achieve a narrow null zone and a precise setting. This method has been termed customized (c)HFP. Selecting the best flicker rate for each patient enables one to customize the variation in flicker sensitivity, which is influenced by variables such as age and disease. ${ }^{36,37}$ If differences among patients in flicker sensitivity are not accounted for (i.e., a fixed flicker frequency is used for each patient), then a patient with low flicker sensitivity (i.e., low critical flicker fusion frequency $[\mathrm{CFF}]$ ) will most likely experience a large null flicker zone. Although the patient may be able to complete the task by eliminating flicker from the test target, the settings are likely to be variable, and patients may exhibit systematic bias toward one end of the null range, resulting in either over- or under-estimation of MPOD. Alternatively, a patient with a high CFF may not be able to eliminate flicker from the test target, which would make the task difficult to complete. As described by Snodderly et al. ${ }^{38}$ the flicker sensitivity issue can be addressed by introducing, as a preliminary test, a CFF task using a single-wavelength band outside the absorption band of MP. Based on an individual's CFF, the optimal HFP flicker frequency is estimated, which facilitates good patient performance and reduces measurement error. An algorithm developed by Nolan and Stringham ${ }^{34}$ was used to estimate optimal HFP flicker frequencies for each retinal locus, including the reference locus. This algorithm (based on a patient's CFF) produced the following predicted flicker frequencies: $0.5^{\circ}$ $=\mathrm{CFF}-6 ; 7^{\circ}=\mathrm{CFF}-12 ; 1^{\circ}=\mathrm{CFF}-6 ; 1.75^{\circ}=\mathrm{CFF}-7 ;$ and $0.25^{\circ}$ $=\mathrm{CFF}-7$. Overall, the values produced by this algorithm yielded low variability in patient settings. The optimization of HFP flicker rate is particularly important in older patients (such as those recruited into this trial), who often demonstrate a significant reduction in their temporal visual sensitivity. ${ }^{37,39}$

An additional methodological consideration involves a test stimulus configuration in which the radiances of the two alternating components are inverse-yoked. In other words, when a patient adjusts the luminance of the blue component to a more intense level, the luminance of the green component is commensurately decreased, and vice versa. This procedure keeps the overall brightness of the test stimulus constant. This approach eliminates the potential distraction caused by changes in brightness experienced by some patients when performing the task in the unyoked setting. This aspect of cHFP is not customized by the experimenter, but is automatically customized for each patient because their settings reflect their own ocular absorption and retinal sensitivity.
MPOD values reported refer to average MPOD across the retina for all loci measured $\left(0.25^{\circ}, 0.5^{\circ}, 1^{\circ}\right.$, and $1.75^{\circ}$ of retinal eccentricity), unless specifically stated.

\section{Statistical Analysis}

Statistical software (SPSS, ver. 15; SPSS Inc., Chicago, IL) was used for analysis and another program was used for graphic presentations (SigmaPlot, ver. 8.0; SPSS Inc.). All variables investigated exhibited a typical normal distribution. Results, expressed as the mean $\pm \mathrm{SD}$, are presented in the text. We conducted repeated-measures analysis of average MPOD across the retina, measured at each of five study visits with a general linear model approach, with lens type as a betweenpatients factor. Differences between two time points, within patients, were assessed using the paired-samples $t$-test. Pearson correlation coefficient analyses were conducted to investigate the relationship between bivariables. We used the $5 \%$ level of significance throughout our analysis.

\section{Results}

\section{Baseline Findings: V1}

Patients. Forty-two patients were recruited. The patients were randomized to receive either the AIOL $(n=21)$ or the ANIOL $(n=21)$ implant as a lens replacement in their cataract surgery. Of the 42 patients recruited, 30 attended all study visits ( 1 week before surgery, 1 week after surgery, and 3, 6, and 12 months after surgery: V1, V2, V3, V4, and V5, respectively). One patient from the AIOL group withdrew after V1, two after V2, and two after V4 ( $n=5$ withdrawals in total). Three patients from the ANIOL group withdrew after V1, two after V2, and two after V4 ( $n=7$ withdrawals in total). The reasons for withdrawal were as follows: patient illness (nonocular); patient deceased; logistics of transport; and not interested in participating further.

Age. The mean age of the patients recruited into the study was $73 \pm 11$ years. The mean age of the patients recruited into the AIOL arm was $71 \pm 11$ years, whereas the mean age of the patients recruited into the ANIOL arm was $74 \pm 11$ years $(P=$ $0.370)$.

Sex. Twenty-five of the patients recruited into the study were men and 17 were women. In the AIOL group, 13 of the patients were men and 8 were women, whereas in the ANIOL group, 12 were men and 9 were women.

Body Mass Index. The mean BMI was $27 \pm 5$ in the entire study group, $29 \pm 5$ in the AIOL group, and $25 \pm 4$ in the ANIOL group $(P=0.017)$.

Diet. As described earlier, each patient was assigned a dietary score representing his/her overall dietary intake of foods containing the macular carotenoids. For the entire study group, the mean diet score was $9.7 \pm 3.6$ of a possible 25 , for the AIOL arm it was $8.8 \pm 3.3$, and for the ANIOL arm it was $10.7 \pm 3.8(P=0.132)$.

Serum $L$ and $Z$. The mean serum concentration of $L$ at V1 in the entire study group was $0.274 \pm 0.095 \mu \mathrm{g} / \mathrm{mL}$, in those in the AIOL arm it was $0.088 \pm 0.013 \mu \mathrm{g} / \mathrm{mL}$, and in those in the ANIOL arm it was $0.103 \pm 0.016 \mu \mathrm{g} / \mathrm{mL}(P=0.505)$. The mean serum concentration of $\mathrm{Z}$ at $\mathrm{V} 1$ was $0.013 \pm 0.011$ $\mu \mathrm{g} / \mathrm{mL}$ in the entire study group, $0.013 \pm 0.002 \mu \mathrm{g} / \mathrm{mL}$ in the AIOL $\mathrm{arm}$, and $0.014 \pm 0.002 \mu \mathrm{g} / \mathrm{mL}$ in the ANIOL $\operatorname{arm}(P=$ $0.785)$.

MPOD. The mean MPOD at V1 averaged across the retina (i.e., average of MPOD at $0.25^{\circ}, 0.5^{\circ}, 1^{\circ}$, and $1.75^{\circ}$ ) was $0.18 \pm$ 0.12 in the entire study group, $0.18 \pm 0.12$ in the AIOL arm, and $0.17 \pm 0.12$ in the ANIOL $\operatorname{arm}(P=0.898)$. The mean MPOD at $\mathrm{V} 1$ at $0.25^{\circ}$ (i.e., peak value) was $0.29 \pm 0.16$ in the entire study group, $0.27 \pm 0.14$ in the AIOL arm, and $0.28 \pm$ 0.17 in the ANIOL $\operatorname{arm}(P=0.870)$. The mean MPOD at V1 at 
TABLE 1. Foveal $\left(0.25^{\circ}\right)$ and Parafoveal $\left(7^{\circ}\right)$ Relative Radiance and Derived MPOD before and after Cataract Surgery in Subjects with the AIOL or the ANIOL Lens Implant

\begin{tabular}{|c|c|c|c|c|c|c|c|}
\hline Subject & $\begin{array}{l}\text { V1 Mean F } \\
\text { Radiance }\end{array}$ & $\begin{array}{l}\text { V1 Mean PF } \\
\text { Radiance }\end{array}$ & $\begin{array}{l}\text { V2 Mean F } \\
\text { Radiance }\end{array}$ & $\begin{array}{l}\text { V2 Mean PF } \\
\text { Radiance }\end{array}$ & $\begin{array}{l}\text { MPOD at } \\
0.25 \mathrm{~V} 1\end{array}$ & $\begin{array}{l}\text { MPOD at } \\
0.25 \mathrm{~V} 2\end{array}$ & $\begin{array}{c}\text { MPOD } \\
\text { Change }\end{array}$ \\
\hline \multicolumn{8}{|l|}{ AIOL } \\
\hline 2 & 1432 & 1145 & 1082 & 840 & 0.18 & 0.18 & 0 \\
\hline 5 & 1778 & 853 & 1475 & 617 & 0.59 & 0.62 & -0.03 \\
\hline 7 & 1624 & 1174 & 1433 & 1095 & 0.28 & 0.22 & 0.06 \\
\hline 11 & 1179 & 1095 & 909 & 730 & 0.06 & 0.14 & -0.08 \\
\hline 12 & 1629 & 1482 & 1114 & 1047 & 0.09 & 0.05 & 0.04 \\
\hline 14 & 2180 & 1323 & 1641 & 830 & 0.55 & 0.54 & 0.01 \\
\hline 16 & 1741 & 1261 & 1449 & 796 & 0.30 & 0.46 & -0.16 \\
\hline 18 & 1295 & 879 & 1191 & 839 & 0.28 & 0.25 & 0.03 \\
\hline 21 & 2121 & 1571 & 1215 & 648 & 0.36 & 0.43 & -0.07 \\
\hline 24 & 1199 & 462 & 1158 & 474 & 0.62 & 0.60 & 0.02 \\
\hline 27 & 1736 & 1350 & 1276 & 829 & 0.24 & 0.31 & -0.07 \\
\hline 29 & 1276 & 899 & 1277 & 993 & 0.26 & 0.21 & 0.05 \\
\hline 31 & 1392 & 787 & 1411 & 712 & 0.42 & 0.47 & -0.05 \\
\hline 32 & 1364 & 1027 & 979 & 759 & 0.22 & 0.17 & 0.05 \\
\hline 33 & 1162 & 915 & 1047 & 831 & 0.17 & 0.16 & 0.01 \\
\hline 34 & 1408 & 974 & 939 & 709 & 0.28 & 0.18 & 0.1 \\
\hline 42 & 1204 & 817 & 984 & 630 & 0.27 & 0.29 & -0.02 \\
\hline Mean & 1513 & 1060 & 1211 & 787 & 0.30 & 0.31 & -0.01 \\
\hline SD & 316 & 282 & 214 & 159 & 0.16 & 0.18 & 0.06 \\
\hline \multicolumn{8}{|l|}{ ANIOL } \\
\hline 3 & 2288 & 1576 & 2143 & 1383 & 0.48 & 0.49 & -0.01 \\
\hline 6 & 1858 & 1199 & 1727 & 1089 & 0.40 & 0.41 & -0.01 \\
\hline 8 & 1689 & 1415 & 1488 & 1185 & 0.17 & 0.19 & -0.02 \\
\hline 9 & 2216 & 2068 & 1500 & 1388 & 0.11 & 0.07 & 0.04 \\
\hline 10 & 1455 & 1199 & 1355 & 1161 & 0.16 & 0.12 & 0.04 \\
\hline 17 & 1181 & 773 & 1146 & 761 & 0.30 & 0.28 & 0.02 \\
\hline 19 & 1819 & 740 & 1651 & 881 & 0.72 & 0.50 & 0.22 \\
\hline 20 & 1183 & 1049 & 1197 & 1060 & 0.09 & 0.09 & 0 \\
\hline 22 & 1526 & 1259 & 1274 & 1043 & 0.17 & 0.15 & 0.02 \\
\hline 23 & 1492 & 1343 & 1331 & 1188 & 0.09 & 0.09 & 0 \\
\hline 26 & 1960 & 1467 & 2122 & 1314 & 0.31 & 0.52 & -0.21 \\
\hline 37 & 1432 & 1015 & 1431 & 980 & 0.27 & 0.29 & -0.02 \\
\hline 40 & 1785 & 1272 & 1770 & 1329 & 0.32 & 0.27 & 0.05 \\
\hline 41 & 1577 & 1004 & 1569 & 971 & 0.37 & 0.39 & -0.02 \\
\hline 43 & 1965 & 1560 & 1505 & 1129 & 0.26 & 0.24 & 0.02 \\
\hline Mean & 1695 & 1263 & 1547 & 1124 & 0.28 & 0.27 & 0.01 \\
\hline SD & 333 & 338 & 298 & 183 & 0.17 & 0.16 & 0.08 \\
\hline
\end{tabular}

$0.5^{\circ}$ was $0.23 \pm 0.15$ for the entire study group, $0.24 \pm 0.15$ in the AIOL arm, and $0.21 \pm 0.15$ in the ANIOL arm. $(P=0.643)$.

\section{MPOD 1 Week before (V1) and 1 Week after (V2) Cataract Surgery}

The mean relative radiance units obtained for $0.25^{\circ}$ (fovea, F) and $7^{\circ}$ (parafovea, PF), along with MPOD for $0.25^{\circ}$, and the change in MPOD between $\mathrm{V} 1$ and $\mathrm{V} 2$ are presented in Table 1 . The data show that MPOD recorded 1 week after cataract surgery was unchanged when compared with readings taken 1 week before surgery in eyes with an AIOL implant (mean MPOD at $0.25^{\circ}, 0.30 \pm 0.16$ and $0.31 \pm 0.18$, for V1 and V2, respectively; mean difference, $-0.01 \pm 0.06$; paired samples $t$-test, $P=0.631$ ) and in eyes with an ANIOL implant (mean MPOD at $0.25^{\circ}, 0.28 \pm 0.17$ and $0.27 \pm 0.16$, for V1 and V2, respectively; mean difference, $0.01 \pm 0.08$; paired samples $t$-test, $P=0.719$ ). In contrast, the F and PF values used in the derivation of MPOD were significantly lower after cataract surgery in those with an AIOL implant (mean \pm SD F and PF radiance units, $1513 \pm 316$ [FV1] and $1211 \pm 214$ [FV2], paired samples $t$-test, $P=0.000$; and $1060 \pm 282[\mathrm{PFV} 1]$ and $787 \pm 159$ [PFV2], paired samples $t$-test, $P=0.000$ ) and in those with an ANIOL implant (mean \pm SD F and PF radiance units $=1695 \pm 333$ [FV1] and $1527 \pm 298$ [FV2], paired samples $t$-test, $P=0.017$, and
$1263 \pm 338$ [PFV1] and $1124 \pm 183$ [PFV2], paired samples $t$-test, $P=0.02$ ) (Table 1 ).

\section{MPOD Alterations for AIOL and ANIOL Groups over the Study Period: V1-V5}

We conducted a repeated-measures analysis of average MPOD across the retina, measured at each of five study visits using a general linear model approach, with lens as a between-patients factor. This resulted in a statistically significant time/lens interaction effect, which remained significant $(P<0.05)$ using any of the standard corrections for violation of sphericity. It is clear from the means plots in Figure 1 and MPOD values presented in Table 2, how this significant time-lens interaction effect arises: MPOD increased with time (at least for some patients) in the ANIOL group, but remained virtually static in the AIOL group.

As the AIOL and ANIOL groups differ significantly with respect to BMI, we conducted further repeated measures analyses with BMI included as a covariate. When comparing average MPOD differences between visits 2 and 5 (i.e., comparing average MPOD immediately after surgery and 12 months later), we obtained $P=0.008$ for the time-IOL type interaction, controlling for BMI. Including all five time points in the analysis, however, the time-IOL type interaction was no longer significant (e.g., $P=0.108$, with Huynh-Feldt correction for 


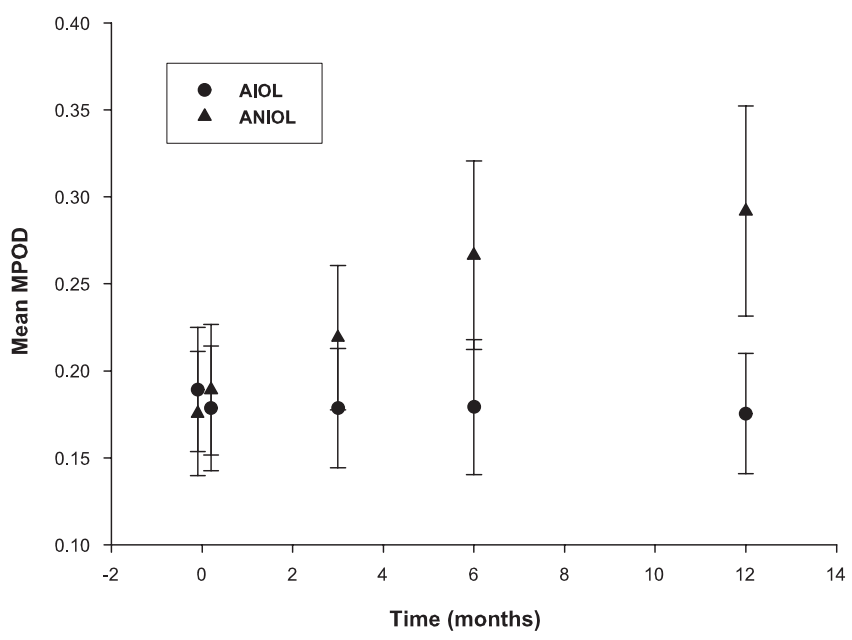

FIGURE 1. Mean MPOD across the retina, at all study visits, in patients in the AIOL and ANIOL groups. Mean MPOD measured at $0.25^{\circ}, 0.5^{\circ}$, $1.0^{\circ}$, and $1.75^{\circ}$ of retinal eccentricity. Standard error bars are included at each time point for both the AIOL and ANIOL groups.

sphericity). Of note, the time-BMI interaction effect was also not significant, indicating that differences in BMI between the AIOL and ANIOL groups remained stable over time. The sensible conclusion to draw from our results therefore appears to be that there is no need to control for BMI differences between the two IOL groups.

Of interest, seven subjects (six in the ANIOL group and one in the AIOL group) showed an increase of 0.08 units or more
TABle 3. Difference in Average MPOD between Visits 2 and 5, at Each Degree of Retinal Eccentricity, in Subjects with the AIOL or the ANIOL Lens Implant

\begin{tabular}{lccccc}
\hline & \multicolumn{2}{c}{ AIOL } & & \multicolumn{2}{c}{ ANIOL } \\
\cline { 2 - 3 } \cline { 5 - 6 } $\begin{array}{c}\text { MPOD } \\
\text { Eccentricity }\end{array}$ & $\begin{array}{c}\text { Mean Difference } \\
\text { (V5 - V2) }\end{array}$ & $\boldsymbol{P}$ & & $\begin{array}{c}\text { Mean Difference } \\
\text { (V5 - V2) }\end{array}$ & $\boldsymbol{P}$ \\
\hline 0.25 & 0.002 & 0.898 & & 0.16 & 0.02 \\
0.5 & -0.017 & 0.393 & & 0.123 & 0.02 \\
1 & -0.008 & 0.167 & & 0.088 & 0.067 \\
1.75 & -0.0045 & 0.703 & & 0.035 & 0.289 \\
\hline
\end{tabular}

$\mathrm{V} 5$ - V2, change in macular pigment between visits 2 and 5; MPOD eccentricity, the eccentricity at which MPOD was measured; $P$, significance at $P \leq 0.05$ (paired-sample $t$-test).

in average MPOD between visits two and five. Comparing this group of seven with the remaining subjects, we found no significant differences in mean BMI (V1), serum L (V1) serum Z (V1), diet score (V1), or age (V1). Distribution of iris color or sex was also not significantly different $(P>0.05$, for all tests). It appears therefore that the observed changes in average MPOD cannot be ascribed to any of these factors.

We also report some results (difference between V5 and V2 [final 12-month study visit minus time of lens implant]) for MPOD measured at each degree of retinal eccentricity $\left(0.25^{\circ}\right.$, $0.5^{\circ}, 1.0^{\circ}$, and $1.75^{\circ}$ ) in both the AIOL and ANIOL groups (Table 3). It is clear from this table that the significant increases in MPOD over the study period in the ANIOL group arose primarily at the center $\left(0.25^{\circ}\right.$ and $\left.0.5^{\circ}\right)$ of the fovea.

TABLE 2. Average MPOD at Each Study Visit in Subjects with the AIOL or the ANIOL Lens Implant and Differences in MPOD between Selected Study Visits

\begin{tabular}{|c|c|c|c|c|c|c|c|c|}
\hline Subject & V1 & V2 & $\mathbf{V 3}$ & V4 & V5 & $\mathbf{v} 3-\mathbf{v} 2$ & V4 - V2 & $v 5-v 2$ \\
\hline \multicolumn{9}{|l|}{ AIOL } \\
\hline 2 & 0.09 & 0.07 & 0.11 & 0.07 & 0.07 & 0.04 & 0 & 0 \\
\hline 5 & 0.4 & 0.45 & 0.38 & 0.43 & 0.4 & -0.07 & -0.02 & -0.05 \\
\hline 7 & 0.3 & 0.17 & 0.14 & 0.11 & 0.12 & -0.03 & -0.06 & -0.05 \\
\hline 11 & 0.03 & 0.07 & 0.06 & 0.02 & 0.03 & -0.01 & -0.05 & -0.04 \\
\hline 12 & 0.05 & 0.01 & 0 & 0.01 & 0 & -0.01 & 0 & -0.01 \\
\hline 14 & 0.43 & 0.38 & 0.38 & 0.42 & 0.37 & 0 & 0.04 & -0.01 \\
\hline 16 & 0.21 & 0.27 & 0.29 & 0.32 & 0.26 & 0.02 & 0.05 & -0.01 \\
\hline 18 & 0.18 & 0.13 & 0.19 & 0.19 & 0.25 & 0.06 & 0.06 & 0.12 \\
\hline 21 & 0.33 & 0.28 & 0.3 & 0.27 & 0.27 & 0.02 & -0.01 & -0.01 \\
\hline 27 & 0.14 & 0.13 & 0.09 & 0.09 & 0.11 & -0.04 & -0.04 & -0.02 \\
\hline 29 & 0.16 & 0.1 & 0.07 & 0.15 & 0.12 & -0.03 & 0.05 & 0.02 \\
\hline 32 & 0.12 & 0.13 & 0.12 & 0.12 & 0.16 & -0.01 & -0.01 & 0.03 \\
\hline 34 & 0.15 & 0.13 & 0.15 & 0.13 & 0.12 & 0.02 & 0 & -0.01 \\
\hline Mean & 0.20 & 0.18 & 0.18 & 0.18 & 0.18 & 0.00 & 0.00 & 0.00 \\
\hline SD & 0.13 & 0.13 & 0.12 & 0.14 & 0.12 & 0.04 & 0.04 & 0.04 \\
\hline \multicolumn{9}{|l|}{ ANIOL } \\
\hline 3 & 0.28 & 0.29 & 0.3 & 0.36 & 0.39 & 0.01 & 0.07 & 0.1 \\
\hline 6 & 0.36 & 0.21 & 0.32 & 0.34 & 0.34 & 0.11 & 0.13 & 0.13 \\
\hline 8 & 0.09 & 0.12 & 0.3 & 0.43 & 0.45 & 0.18 & 0.31 & 0.33 \\
\hline 9 & 0.03 & 0.02 & 0.01 & 0.01 & 0 & -0.01 & -0.01 & -0.02 \\
\hline 10 & 0.12 & 0.1 & 0.07 & 0.07 & 0.06 & -0.03 & -0.03 & -0.04 \\
\hline 17 & 0.22 & 0.25 & 0.28 & 0.17 & 0.25 & 0.03 & -0.08 & 0 \\
\hline 19 & 0.37 & 0.43 & 0.28 & 0.38 & 0.52 & -0.15 & -0.05 & 0.09 \\
\hline 20 & 0.04 & 0.06 & 0.07 & 0.15 & 0.08 & 0.01 & 0.09 & 0.02 \\
\hline 22 & 0.12 & 0.17 & 0.26 & 0.36 & 0.47 & 0.09 & 0.19 & 0.3 \\
\hline 26 & 0.13 & 0.32 & 0.44 & 0.58 & 0.54 & 0.12 & 0.26 & 0.22 \\
\hline 40 & 0.17 & 0.11 & 0.08 & 0.08 & 0.11 & -0.03 & -0.03 & 0 \\
\hline Mean & 0.18 & 0.19 & 0.22 & 0.27 & 0.29 & 0.03 & 0.08 & 0.10 \\
\hline SD & 0.12 & 0.12 & 0.14 & 0.18 & 0.20 & 0.09 & 0.13 & 0.13 \\
\hline
\end{tabular}

V3 - V2 $=$ change in MPOD between visit 2 and visit 3 (AIOL: $P=0.964$; ANIOL: $P=0.392$ )

$\mathrm{V} 4-\mathrm{V} 2=$ change in MPOD between visit 2 and visit 4 (AIOL: $P=1.000$; ANIOL: $P=0.050$ )

$\mathrm{V} 5-\mathrm{V} 2=$ change in MPOD between visit 2 and visit 5 (AIOL: $P=0.474$; ANIOL: $P=0.028$ ) 


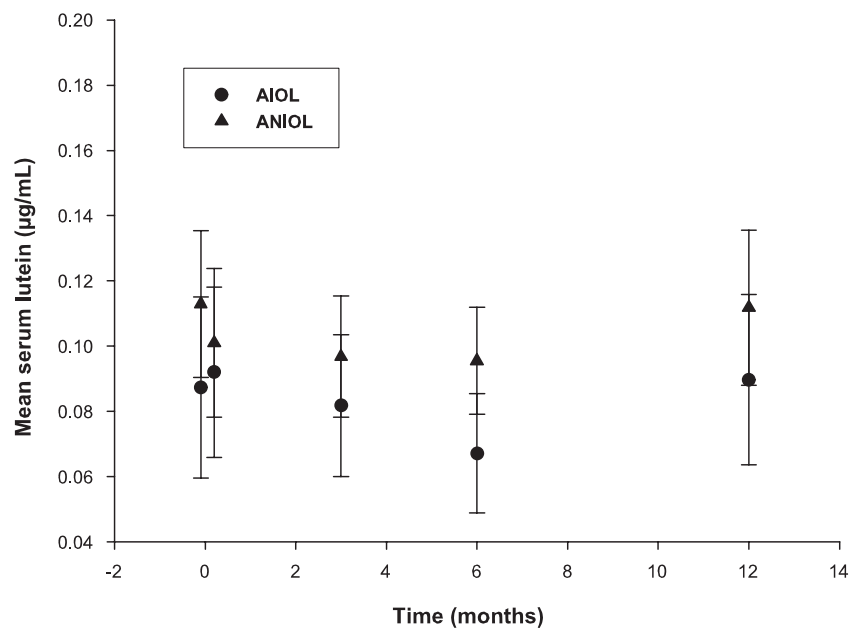

FIGURE 2. Mean serum concentrations of $\mathrm{L}$, at all study visits, in patients in the AIOL and ANIOL groups. Mean serum L is the mean concentration of $\mathrm{L}$ at each study visit. Standard error bars are included at each time point for both the AIOL and ANIOL groups.

\section{Serum $L$ and $Z$ Concentrations for AIOL and ANIOL Groups over the Study Period: V1-V5}

No significant serum L effects were observed over the study period (Fig. 2). There was a significant time effect for serum $\mathrm{Z}$ over the study period (repeated-measures, general linear model, e.g., using Huynh-Feldt correction for sphericity, $P=$ $0.038)$, but not a significant time-IOL interaction $(P>0.05$ for all tests) (Fig. 3). Thus, serum $\mathrm{Z}$ was significantly different at different time points, but this was true in both the AIOL and ANIOL groups.

\section{Visual Acuity}

Visual acuity was assessed using a Bailey-Lovie distance chart and recorded with $\log$ MAR notation. For statistical analysis, logMAR acuity was converted to a visual acuity rating (VAR) notation. LogMAR 0 acuity (equivalent to 20/20 Snellen acuity) was assigned a score of 100. Acuity was calculated on the basis of every letter that was correctly identified above or below this line, with each letter worth one mark, and each line therefore, five marks. For example, a subject with corrected logMAR acuity of 0.3 (Snellen equivalent 20/40), was assigned a mark of $85[100-15$ (3 lines $=15$ marks) $]$. If the subject could correctly identify two additional letters on the smaller logMAR 0.2 line, they were assigned a score of 87 .

The mean BCVAR for the entire study group at V1 was $82.49 \pm 7.68$. There was a predictable and statistically significant improvement in acuity after cataract surgery and postoperative BCVAR at V2 for the entire study group was $89.69 \pm$ 6.79 (paired samples $t$-test; $t=-4.703, P<0.001$ ). The improvement in acuity was maintained throughout the study period, and BCVAR for the entire study group at V5 was $91.84 \pm 5.20$.

Visual acuity results were further analyzed to assess the impact of lens type on visual outcome. The mean BCVAR at V1 was $82.57 \pm 8.37$ for the AIOL group, and 82.39 \pm 7.02 for the ANIOL group. Postoperative BCVAR at V2 was $89.72 \pm 6.57$ for the AIOL group and $89.64 \pm 7.32$ for the ANIOL group. Final BCVAR at V5 was $91.93 \pm 6.16$ for the AIOL group and $91.73 \pm$ 4.18 for the ANIOL group. Statistical analysis confirms a significant time effect on BCVAR (repeated-measures, general linear model, e.g., using Huynh-Feldt correction for sphericity $P<$ $0.001)$, but not a significant time-lens interaction $(P>0.05$ for all tests). Similar improvements in acuity were thus observed in the AIOL and ANIOL groups over the time course of the study.

\section{Discussion}

This study was designed to measure and compare the effect of cataract surgery on MPOD over a 1-year period in eyes in which a standard UV-light filtering intraocular lens (AIOL) was implanted and in eyes in which a blue-light and UV-light filtering intraocular lens (ANIOL) was implanted. Forty-two patients scheduled for cataract surgery at WRH were randomized to have either an AIOL or an ANIOL implanted during surgery (in place of the cataractous lens). MPOD was measured at baseline ( 1 week before and 1 week after cataract surgery) and at 3, 6, and 12 months after surgery in all patients. Serum carotenoid concentrations were also measured at each study visit in all patients to control for any potentially confounding variables relating to their MPOD measurements. This study was prospective, randomized, controlled, double-blind in design, and, to our knowledge, it is the first of its kind.

Blue light has been implicated in the development and progression of AMD after cataract surgery. ${ }^{4,22,40-44}$ Indeed, after cataract surgery, the retina is exposed to more blue light than at any stage in the patient's lifetime, ${ }^{41}$ and Wang et al. reported a 5-year, two- to fivefold increased risk of development of late-stage AMD after cataract surgery in individuals without AMD at baseline. ${ }^{22}$ Furthermore, in vitro, both RPE cell apoptosis and upregulation of vascular endothelial growth factor (VEGF), one of the most potent proangiogenic factors in the pathogenesis of neovascular AMD, have been demonstrated in A2E-laden RPE cells on exposure to light, ${ }^{40,42}$ but these effects are relatively attenuated when the light is filtered through a blue-light filtering IOL. ${ }^{43,44}$ Of interest, the human crystalline lens yellows with age, and transmits less blue light than a younger crystalline lens. ${ }^{24}$ The transmission of blue light is further reduced with cataract formation, and this may confer some degree of protection against AMD at a stage in life when the concentration of RPE lipofuscin (the major chromophore in this line of cells) peaks at the macula. ${ }^{45}$ If indeed cataract surgery is an independent risk factor for development or progression of AMD, replacement of the natural (blue light filtering) crystalline lens with a clear (non-blue light filtering) artificial IOL may contribute to any observed increase in risk for progression or development of AMD after cataract surgery.

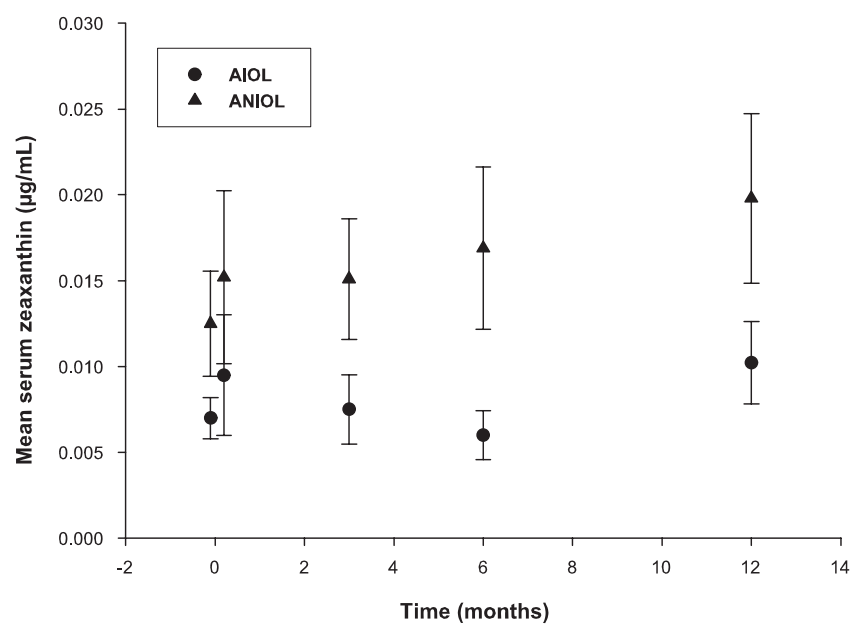

Figure 3. Mean serum concentrations of $Z$, at all study visits, in patients in the AIOL and ANIOL groups. Mean serum $Z$ is the mean concentration of $Z$ at each study visit. Standard error bars are included at each time point for both the AIOL and ANIOL groups. 
Alcon has been producing a blue-light-filtering intraocular lens (ANIOL) since 2000, with a view to attenuating any increased risk of progression or development of AMD after cataract surgery. The ANIOL was the first blue-light-filtering IOL on the market in North America, ${ }^{46}$ and was designed to mimic the transmittance characteristics of the adult human crystalline lens, with the absorption characteristics of a 20-D ANIOL being similar to that of a 53-year-old crystalline lens. ${ }^{47}$ The ANIOL is entirely similar to the AIOL apart from a covalently bound chromophore that partially absorbs light in the 400- to 500-nm spectral range. Conventional UV-blocking IOLs (such as the AIOL) display a sharp increase in light transmission beyond 400 $\mathrm{nm}$, whereas the ANIOL allows only a 10\% transmittance at 406 $\mathrm{nm}$ increasing to a $50 \%$ transmittance at $459 \mathrm{~nm}$, and an $80 \%$ transmittance at $500 \mathrm{~nm},{ }^{23}$ thus blocking transmission of a large proportion of high-energy and potentially injurious shortwavelength light. Despite the greatly reduced transmission of short-wavelength (blue) light, visual acuity, color perception, and contrast sensitivity have repeatedly been found to be equivalent under photopic and mesopic conditions with the UV-only blocking AIOL and the ANIOL. ${ }^{48-59}$ The acuity results reported herein are in general agreement with those in previous studies and provide further evidence that lens type, whether ANIOL or AIOL, has little impact on postoperative photopic, high-contrast visual acuity. Reduced scotopic sensitivity has been demonstrated in some studies with the $\mathrm{ANIOL}^{47,60,61}$; however, this has generally been accepted to be of little visual or functional significance ${ }^{61-65}$ and does not affect patients' quality of life. ${ }^{66}$

In this study, we set out to evaluate the effect of implantation of a blue-light filtering IOL on MPOD and to compare the findings with control subjects in whom a non-blue-light-filtering IOL was implanted. Given the growing, but inconclusive, evidence base for a protective role of MP in AMD (recently reviewed by Loane et al $^{67}$ ), the results of this study indicate that implantation of an ANIOL at the time of cataract surgery may confer protection against progression or development of AMD. We postulated that reducing the amount of blue light incident on the retina (by implantation of an ANIOL as opposed to an AIOL) would lead to less generation of free radicals in response to irradiation with short-wavelength (blue) light. ${ }^{68}$ As a consequence, in theory at least, depletion of MP over time (caused by neutralizing free radicals) would be attenuated in those eyes with an ANIOL implant compared with those with an AIOL implant.

MPOD measured 1 week after surgery was unchanged compared with readings taken 1 week before surgery in each study group (although foveal and parafoveal radiances were reduced after surgery as expected, but the relative radiance remained consistent). The ANIOL more closely approximates the transmittance characteristics of the natural crystalline lens and, as such, reduced radiances were more evident in the AIOL group. The stability of MPOD measured before and after surgery demonstrates that the measurement of MPOD using HFP is not influenced artifactually or otherwise by the event of cataract surgery (whether an AIOL or an ANIOL is implanted), and this stability therefore lends validity to MPOD measurements taken at 3, 6, and 12 months after surgery. These findings are consistent with those of Ciulla et al., ${ }^{69}$ who also found no significant change in MPOD measurements taken immediately before and after cataract surgery in a patient population with inclusion and exclusion criteria similar to those in our study (with no patients in their study having any evidence of macular disease and a median best corrected Snellen distance visual acuity before surgery of 20/50).

We found that MPOD remained stable over the course of the study in the group with an AIOL implant. However, and in contrast, in eyes in which an ANIOL was implanted, MPOD increased over the duration of the study period, with a statistically significant increase in MPOD at months 3, 6, and 12 after surgery. Of note, dietary levels and serum concentrations of $\mathrm{L}$ and $\mathrm{Z}$ remained largely unchanged over the course of the study. Nolan et al. ${ }^{70}$ have demonstrated serial month-to-month consistency of MPOD measurements using HFP over a 24month period in healthy subjects in whom serum concentrations of the macular carotenoids were stable, consistent with the findings of Wenzel et al. ${ }^{71}$ (investigating MPOD in two male subjects over a 20-day period), who reported that the optical density of the macular carotenoids is unaffected by diurnal variations in exposure to ambient levels of light. Of note, Wenzel et al. and Nolan et al. ${ }^{70}$ were uncontrolled observational studies. In other words, the stability of MPOD appears to be unaffected (in the context of a stable diet) over long periods, which renders our findings all the more interesting, given that we have (in the context of a randomized controlled trial) demonstrated that differential filtration of blue light after cataract surgery influences MPOD with the passage of time. ${ }^{70,71}$

Our findings prompt a discussion on the relationship, if any, between MPOD and age, as the yellowing of the crystalline lens with age results in an age-related increase in prereceptoral filtration of blue light. This phenomenon, given our finding that blue-filtering IOLs result in augmentation of MPOD, in theory at least, might be expected to give rise to an age-related increase in MPOD. However, most studies do not support such an association, perhaps reflecting the known age-related increase in oxidant load, which may offset any protective effect of prereceptorial filtration of blue light. ${ }^{3}$

Our initial hypothesis postulated a reduction in MPOD with implantation of an AIOL (due to the increased transmittance of short-wavelength blue light) and relative stabilization of MPOD with ANIOL implantation and, as such, our findings were somewhat unexpected. The MPOD in those eyes with the AIOL implant remained constant over the one-year study period, despite the fact that blue-light transmission with the AIOL implant is greater than that in a 71-year-old (mean age of patients in the AIOL group) cataractous lens. Indeed, a 74-yearold (mean age of patients in the ANIOL group) cataractous lens may also be expected to transmit less blue light than the ANIOL. However, in eyes with an ANIOL implant, MPOD increased over the 1-year follow-up period in our study. It is difficult to explain our findings, although it is possible that increased visible light irradiation of the retina after cataract surgery stimulates enhanced retinal capture of circulating $\mathrm{L}$ and $\mathrm{Z}$ in an attempt to offset a possible increase in photooxidative retinal injury, but that the heralded increase in photooxidative retinal injury does not occur in eyes with a bluefiltering IOL implant. Also, it is possible that there is some degree of autoregulation of MPOD in the context of a stable diet and that such autoregulation is disturbed by cataract surgery as a result of increased light incident on the macula and a consequential depletion of existing MP (because of increased photochemical oxidant load). However, the presence of a blue-filtering IOL attenuates the amount of blue light incident on the retina, with perhaps a parallel reduction in the extent of depletion of macular carotenoids (because of reduced photochemical injury), with a consequential rise in MPOD.

In conclusion, this study provides evidence that implanting an IOL that filters blue light (ANIOL) results in augmentation of MPOD. The importance of this finding rests on the fact that any benefits associated with augmentation of MPOD, in terms of AMD prevention or progression (yet to be proven), will be conferred on patients with an ANIOL implant at the time of cataract surgery, and may be of particular importance in the modern era when IOL implantation often occurs at an earlier stage in a patient's lifetime (such as in pediatric cataract surgery, refractive lens exchange and relatively early lens opacity 
in patients with a long postoperative life expectancy). However, further study is required in the form of controlled longterm trials to investigate whether implantation of a blue-light filtering IOL is effective in preventing or delaying development or progression of AMD.

\section{References}

1. Friedman DS, O'Colmain BJ, Munoz B, et al. Prevalence of agerelated macular degeneration in the United States. Arch Ophthalmol. 2004;122(4):564-572.

2. Resnikoff S, Pascolini D, Etya'ale D, et al. Global data on visual impairment in the year 2002. Bull World Health Organ. 2004; 82(11):844-851.

3. Beatty S, Koh HH, Henson D, Boulton M. The role of oxidative stress in the pathogenesis of age-related macular degeneration. Surv Ophthalmol. 2000;45(2):115-134.

4. Algvere PV, Marshall J, Seregard S. Age-related maculopathy and the impact of blue light hazard. Acta Ophthalmol Scand. 2006; 84(1):4-15.

5. Dillon J, Zheng L, Merriam JC, Gaillard ER. Transmission of light to the aging human retina: possible implications for age related macular degeneration. Exp Eye Res. 2004;79(6):753-759.

6. Snodderly DM, Auran JD, Delori FC. The macular pigment. II. Spatial distribution in primate retinas. Invest Ophthalmol Vis Sci. 1984;25(6):674-685.

7. Stringham JM, Hammond BR, Wooten BR, Snodderly DM. Compensation for light loss resulting from filtering by macular pigment: relation to the S-cone pathway. Optom Vis Sci. 2006;83(12):887894.

8. Junghans A, Sies H, Stahl W. Macular pigments lutein and zeaxanthin as blue light filters studied in liposomes. Arch Biochem Biophys. 2001;391(2):160-164.

9. Trieschmann M, van Kuijk FJ, Alexander R, et al. Macular pigment in the human retina: histological evaluation of localization and distribution. Eye. 2008;22(1):132-137.

10. Liu IY, White L, LaCroix AZ. The association of age-related macular degeneration and lens opacities in the aged. Am J Public Health. 1989;79(6):765-769.

11. Krishnaiah S, Das T, Nirmalan PK, et al. Risk factors for age-related macular degeneration: findings from the Andhra Pradesh eye disease study in South India. Invest Ophthalmol Vis Sci. 2005;46(12): 4442- 4449

12. Buch H, Vinding T, la Cour M, Jensen GB, Prause JU, Nielsen NV. Risk factors for age-related maculopathy in a 14-year follow-up study: the Copenhagen City Eye Study. Acta Ophthalmol Scand. 2005;83(4):409-418.

13. Klein R, Klein BE, Wong TY, Tomany SC, Cruickshanks KJ. The association of cataract and cataract surgery with the long-term incidence of age-related maculopathy: the Beaver Dam Eye Study. Arch Ophthalmol. 2002;120(11):1551-1558.

14. West SK, Rosenthal FS, Bressler NM, et al. Exposure to sunlight and other risk factors for age-related macular degeneration. Arch Ophthalmol. 1989;107(6):875-879.

15. Khan JC, Shahid H, Thurlby DA, et al. Age related macular degeneration and sun exposure, iris colour, and skin sensitivity to sunlight. Br J Ophthalmol. 2006;90(1):29-32.

16. Darzins $\mathrm{P}$, Mitchell $\mathrm{P}$, Heller RF. Sun exposure and age-related macular degeneration: an Australian case-control study. Ophthalmology. 1997;104(5):770-776.

17. Tomany SC, Cruickshanks KJ, Klein R, Klein BEK, Knudtson MD. Sunlight and the 10-year incidence of age-related maculopathy: The Beaver Dam Eye Study. Arch Ophthalmol. 2004;122(5):750 757.

18. Cruickshanks KJ, Klein R, Klein BEK. Sunlight and age-related macular degeneration: the Beaver Dam Eye Study. Arch Ophthalmol. 1993;111(4):514-518.

19. Hirakawa M, Tanaka M, Tanaka Y, et al. Age-related maculopathy and sunlight exposure evaluated by objective measurement. $\mathrm{BrJ}$ Ophthalmol. 2008;92(5):630-634.

20. Taylor HR, Munoz B, West S, Bressler NM, Bressler SB, Rosenthal FS. Visible light and risk of age-related macular degeneration.
Trans Am Ophthalmol Soc. 1990;88:163-173; discussion 173178.

21. Fletcher AE, Bentham GC, Agnew M, et al. Sunlight exposure, antioxidants, and age-related macular degeneration. Arch Ophthalmol. 2008;126(10):1396-1403.

22. Wang JJ, Klein R, Smith W, Klein BEK, Tomany S, Mitchell P. Cataract surgery and the 5-year incidence of late-stage age-related maculopathy: pooled findings from the Beaver Dam and Blue Mountains Eye Studies. Ophthalmology. 2003;110(10):19601967.

23. Brockmann C, Schulz M, Laube T. Transmittance characteristics of ultraviolet and blue-light-filtering intraocular lenses. J Cataract Refract Surg. 2008;34(7):1161-1166.

24. Gaillard ER, Zheng L, Merriam JC, Dillon J. Age-related changes in the absorption characteristics of the primate lens. Invest Ophthalmol Vis Sci. 2000;41(6):1454-1459.

25. Bockelbrink A, Roll S, Ruether K, Rasch A, Greiner W, Willich SN. Cataract surgery and the development or progression of age-related macular degeneration: a systematic review. Surv Ophthalmol. 2008;53(4):359-367.

26. Roodenburg AJ, Leenen R, van het Hof KH, Weststrate JA, Tijburg LB. Amount of fat in the diet affects bioavailability of lutein esters but not of $\alpha$-carotene, $\beta$-carotene, and vitamin $\mathrm{E}$ in humans. $A m \mathrm{~J}$ Clin Nutr. 2000;71(5):1187-1193.

27. Unlu NZ, Bohn T, Clinton SK, Schwartz SJ. Carotenoid absorption from salad and salsa by humans is enhanced by the addition of avocado or avocado oil. J Nutr. 2005;135(3):431-436.

28. Snodderly DM, Chung HC, Caldarella SM, Johnson EJ. The influence of supplemental lutein and docosahexaenoic acid on their serum levels and on macular pigment. Invest Ophthalmol Vis Sci. 2005;46(5): 1766 .

29. Wooten BR, Hammond BR, Land RI, Snodderly DM. A practical method for measuring macular pigment optical density. Invest Ophthalmol Vis Sci. 1999;40(11):2481-2489.

30. Hammond BR Jr, Wooten BR, Smollon B. Assessment of the validity of in vivo methods of measuring human macular pigment optical density. Optom Vis Sci. 2005;82(5):387-404.

31. Hammond BR, Wooten BR, Snodderly DM. Individual variations in the spatial profile of human macular pigment. J Opt Soc Am A Opt Image Sci Vision. 1997;14(6):1187-1196.

32. Werner JS, Donnelly SK, Kliegl R. Aging and human macular pigment density: appended with translations from the work of Max Schultze and Ewald Hering. Vision Res. 1987;27:257-268.

33. Hammond BR, Caruso-Avery M. Macular pigment optical density in a southwestern sample. Invest Ophthalmol Vis Sci. 2000;41(6): 1492-1497.

34. Nolan JM, Stringham JM, Beatty S, Snodderly DM. Spatial profile of macular pigment and its relationship to foveal architecture. Invest Ophthalmol Vis Sci. 2008;49(5):2134-2142.

35. Loane E, Stack J, Beatty S, Nolan JM. Measurement of macular pigment optical density using two different heterochromatic flicker photometers. Curr Eye Res. 2007;32(6):555-564.

36. Tyler CW. Two processes control variations in flicker sensitivity over the life span. J Opt Soc Am A. 1989;6(4):481-490.

37. Falsini B, Fadda A, Iarossi G, et al. Retinal sensitivity to flicker modulation: reduced by early age-related maculopathy. Invest Ophthalmol Vis Sci. 2000;41(6):1498-1506.

38. Snodderly DM, Mares JA, Wooten BR, Oxton L, Gruber M, Ficek T. Macular pigment measurement by heterochromatic flicker photometry in older subjects: The carotenoids and age-related eye disease study. Invest Ophthalmol Vis Sci. 2004;45(2):531-538.

39. Phipps JA, Dang TM, Vingrys AJ, Guymer RH. Flicker perimetry losses in age-related macular degeneration. Invest Ophthalmol Vis Sci. 2004; 45(9):3355-3360.

40. Witmer AN, Vrensen GFJM, Van Noorden CJF, Schlingemann RO. Vascular endothelial growth factors and angiogenesis in eye disease. Prog Retin Eye Res. 2003;22(1):1-29.

41. Pollack A, Marcovich A, Bukelman A, Oliver M. Age-related macular degeneration after extracapsular cataract extraction with intraocular lens implantation. Ophthalmology. 1996;103(10):15461554 . 
42. Sparrow JR, Cai B. Blue light-induced apoptosis of A2E-containing RPE: involvement of caspase-3 and protection by $\mathrm{Bcl}-2$. Invest Ophthalmol Vis Sci. 2001;42(6):1356-1362.

43. Yanagi $\mathrm{Y}$, Inoue $\mathrm{Y}$, Iriyama A, Jang WD. Effects of yellow intraocular lenses on light-induced upregulation of vascular endothelial growth factor. J Cataract Refract Surg. 2006;32(9):1540-1544.

44. Sparrow JR, Miller AS, Zhou J. Blue light-absorbing intraocular lens and retinal pigment epithelium protection in vitro. $J$ Cataract Refract Surg. 2004;30(4):873-878.

45. Feeneyburns L, Hilderbrand ES, Eldridge S. Aging human RPE: morphometric analysis of macular, equatorial, and peripheral cells. Invest Ophthalmol Vis Sci. 1984;25(2):195-200.

46. Pierre A, Wittich W, Faubert J, Overbury O. Luminance contrast with clear and yellow-tinted intraocular lenses. J Cataract Refract Surg. 2007;33(7):1248-1252.

47. Mainster MA, Sparrow JR. How much blue light should an IOL transmit? Br J Ophthalmol. 2003;87(12):1523-1529.

48. Cionni RJ, Tsai JH. Color perception with AcrySof natural and AcrySof single-piece intraocular lenses under photopic and mesopic conditions. J Cataract Refract Surg. 2006;32(2):236-242.

49. Mayer S, Wirbelauer C, Pham DT. Functional results after intraocular lens implantation with or without blue light filter: an intraindividual comparison (in German). Klin Monatsbl Augenheilkd. 2006;223(2):142-146

50. Hayashi K, Hayashi H. Visual function in patients with yellow tinted intraocular lenses compared with vision in patients with non-tinted intraocular lenses. Br J Ophthalmol. 2006;90(8):10191023.

51. Landers J, Tan TH, Yuen J, Liu H. Comparison of visual function following implantation of Acrysof Natural intraocular lenses with conventional intraocular lenses. Clin Exp Ophthalmol. 2007; 35(2):152-159.

52. Leibovitch I, Lai T, Porter N, Pietris G, Newland H, Selva D. Visual outcomes with the yellow intraocular lens. Acta Ophthalmol Scand. 2006;84(1):95-99.

53. Barisic A, Dekaris I, Gabric N, et al. Blue light filtering intraocular lenses in phacoemulsification cataract surgery. Coll Antropol. 2007;31(suppl 1):57-60.

54. Niwa K, Yoshino Y, Okuyama F, Tokoro T. Effects of tinted intraocular lens on contrast sensitivity. Ophthalmic Physiol Opt. 1996;16(4):297-302.

55. Muftuoglu O, Karel F, Duman R. Effect of a yellow intraocular lens on scotopic vision, glare disability, and blue color perception. $J$ Cataract Refract Surg. 2007;33(4):658-666.

56. Rodriguez-Galietero A, Montes-Mico R, Munoz G, Barran-Diego C. Comparison of contrast sensitivity and color discrimination after clear and yellow intraocular lens implantation. J Cataract Refract Surg. 2005;31(9):1736-1740.
57. Schmidinger G, Menapace R, Pieh S. Intraindividual comparison of color contrast sensitivity in patients with clear and blue-lightfiltering intraocular lenses. J Cataract Refract Surg. 2008;34(5): $769-773$.

58. Bhattacharjee H, Bhattacharjee K, Medhi J. Visual performance: comparison of foldable intraocular lenses.J Cataract Refract Surg. 2006;32(3):451- 455.

59. Marshall J, Cionni RJ, Davison J, et al. Clinical results of the blue-light filtering AcrySof Natural foldable acrylic intraocular lens. J Cataract Refract Surg. 2005;31(12):2319-2323.

60. Werner JS. Night vision in the elderly: consequences for seeing through a "blue filtering" intraocular lens. Br J Ophthalmol. 2005; 89(11):1518-1521.

61. Schwiegerling J. Blue-light-absorbing lenses and their effect on scotopic vision. J Cataract Refract Surg. 2006;32(1):141-144.

62. Aharonoff GB, Dennis MG, Elshinawy A, Zuckerman JD, Koval KJ. Circumstances of falls causing hip fractures in the elderly. Clin Orthop Relat Res. 1998;(348):10-14.

63. Norton R, Campbell AJ, Lee-Joe T, Robinson E, Butler M. Circumstances of falls resulting in hip fractures among older people. $\mathrm{J} \mathrm{Am}$ Geriatr Soc. 1997;45(9):1108-1112.

64. Vetter NJ, Lewis PA, Ford D. Can health visitors prevent fractures in elderly people? BMJ. 1992;304(6831):888-890.

65. Mainster MA, Timberlake GT. Why HID headlights bother older drivers. Br J Ophthalmol. 2003;87(1):113-117.

66. Espindle D, Crawford B, Maxwell A, et al. Quality-of-life improvements in cataract patients with bilateral blue light-filtering intraocular lenses: clinical trial. J Cataract Refract Surg. 2005;31(10): 1952-1959.

67. Loane E, Kelliher C, Beatty S, Nolan JM. The rationale and evidence base for a protective role of macular pigment in age-related maculopathy. Br J Ophthalmol. 2008;92(9):1163-1168.

68. Rozanowska M, Jarvis-Evans J, Korytowski W, Boulton ME, Burke JM, Sarna T. Blue light-induced reactivity of retinal age pigment: in vitro generation of oxygen-reactive species. J Biol Chem. 1995; 270(32):18825-18830.

69. Ciulla TA, Hammond BR Jr, Yung CW, Pratt LM. Macular pigment optical density before and after cataract extraction. Invest Ophthalmol Vis Sci. 2001;42(6):1338-1341.

70. Nolan JM, Stack J, Mellerio J, Godhinio M, O'Donovan O, Beatty S. Monthly consistency of macular pigment optical density and serum concentrations of lutein and zeaxanthin. Curr Eye Res. 2006; 31(2):199-213.

71. Wenzel AJ, Fuld K, Stringham JM. Light exposure and macular pigment optical density. Invest Ophthalmol Vis Sci. 2003;44(1): 306-309. 